\title{
AUTOMATIC PARKING OF SELF-DRIVING CAR BASED ON LIDAR
}

\author{
Bijun Lee ${ }^{a}$, Yang $\mathrm{Wei}^{\mathrm{a}}$, I. Yuan Guo ${ }^{\mathrm{a}}$ \\ a State Key Laboratory of Information Engineering in Surveying, Mapping and Remote Sensing, Wuhan University, China - \\ (Lee, guoyuan, yangwei)@whu.edu.cn
}

\author{
Commission VI, WG VI/4
}

KEY WORDS: Automatic driving, Parallel driving, Perpendicular driving, Path planning, Fuzzy logic controller

\begin{abstract}
:
To overcome the deficiency of ultrasonic sensor and camera, this paper proposed a method of autonomous parking based on the selfdriving car, using HDL-32E LiDAR. First the 3-D point cloud data was preprocessed. Then we calculated the minimum size of parking space according to the dynamic theories of vehicle. Second the rapidly-exploring random tree algorithm (RRT) algorithm was improved in two aspects based on the moving characteristic of autonomous car. And we calculated the parking path on the basis of the vehicle's dynamics and collision constraints. Besides, we used the fuzzy logic controller to control the brake and accelerator in order to realize the stably of speed. At last the experiments were conducted in an autonomous car, and the results show that the proposed automatic parking system is feasible and effective.
\end{abstract}

\section{INTRODUCTION}

Autonomous vehicle is a hot research area now. And automatic parking as an important part of autonomous vehicle have been concerned by more and more researchers. Besides, as the number of car increases, parking has becoming an increasingly difficult matter. In this case, automatic parking is an indispensable part in our life.

The automatic parking system consists of target position designation, path planning and parking guidance (Wang, Song et al. 2014). The scanning laser radar (Jung, Cho et al. 2008), ultrasonic sensor, (Park, Kim et al. 2008) and binocular (Kaempchen, Franke et al. 2002) are used to gather exteroceptive information and identify the parking spot. However the ultrasonic sensor can't adapt to the situation that the both sides of parking place is empty and there are lots of blind spots when using camera. In order to improve the efficiency of automatic parking, we chose the HDL-32 LiDAR to detect parking spot.

About the path planning and control, there are mainly about two categories(Marouf, Pollard et al. 2014). One is based on the fuzzy logic to transfer human driver's parking skill to automatic parking controller(Laugier and Paromtchik 1996). Yanan Zhao designed novel fuzzy logic controllers using the kinematic model of a skid steering autonomous ground vehicle((Zhao and Jr 2005) And Xiyang Yang designed a fuzzy logic control method combined hierarchical control and variable universe(Yang and You 2010). However it is difficult to model in this way.

The other one is based on vehicle's dynamics and constraints to planning a feasible geometry path in advance, and then the vehicle is controlled to follow the reference path(Min and Choi 2013). Inoue proposed a path planning method composed of control laws for the steering angle and the driving velocity (Liu, Dao et al. 2005). JinZe Song proposed a method to generate collision-free space based on the differential flat theory and spline theory (song 2009). This approach requires high control precision of speed and direction. And it is more appropriate to apply to autonomous car.
In this paper, we choose the second way. And we proved the RRT algorithm to plan parking path.

\section{PREPROCESSING AND CALCULATING}

In order to increase efficiency, the preprocessing and segmentation of 3-D cloud data is necessary since the large amount of data. We extracted the available parking space information by recognizing the geometric feature of stopped car.

\subsection{PREPROCESSING OF DATA}

In china, traffic keeps on the right of the road. We need to find the parking spot on the right side of the car. And the obstacle information on the left side is used to constrain the parking path. However, the data in the distance will interfere the process of information extraction and increase the time and calculating cost. For this reason, we defined the region of interest (ROI). The distance to the left and right of LiDAR are $8 \mathrm{~m}$ and $10 \mathrm{~m}$ and the distance to the forward and backward of LiDAR are $16 \mathrm{~m}$ and $8 \mathrm{~m}$.

We care about the information of parked cars and the obstacles. Ground data and the point data of tree are useless for us. The distance from LiDAR to ground is $2 \mathrm{~m}$. And the tree is taller than LiDAR. So the through filter is used to retain the data $-2 \mathrm{~m}<\mathrm{z}<0 \mathrm{~m}$. There exist safe distance between parked cars and obstacles. Based on this feature, the Euclidean cluster is applied to extract parked cars and obstacles. At last we projected the data to XOY plane and replaced it by minimum bounding rectangle (MBR).

Figure 1(a) shows the original 3D point cloud. Figure 1(b) depicts the extracted ROI. The result of Euclidean cluster is depicted by Figure 1(c). And Figure 1(d) shows the MBR of parked cars and obstacles.

The distance between MBR compared to the minimum size of parking spot can determine the area of parking. 


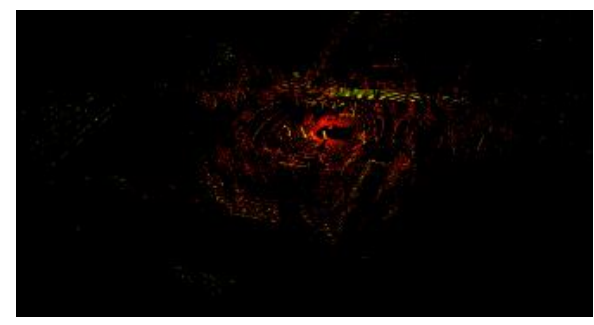

Figure 1(a). Original data

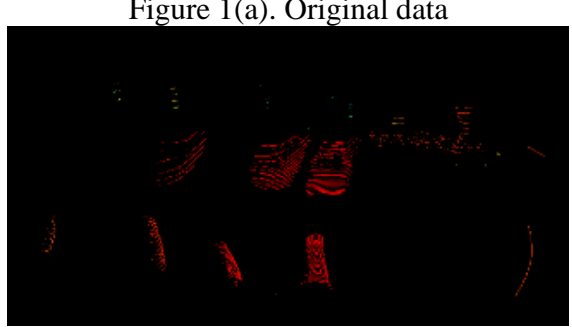

Figure 1(b). Region of interest

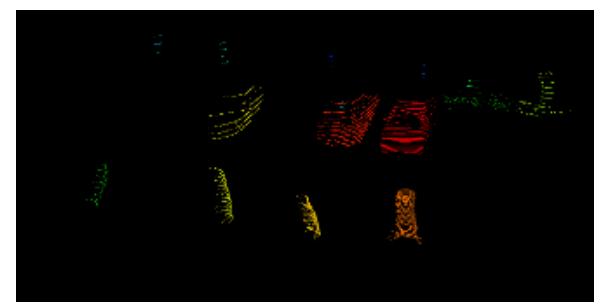

Figure 1(c). Data after extracted

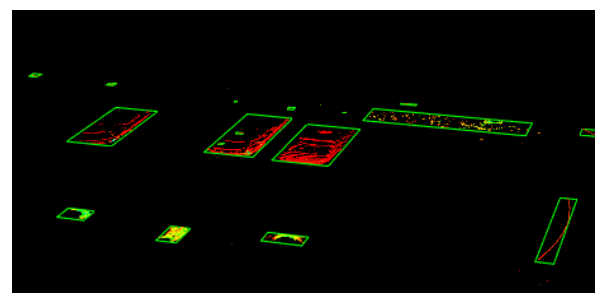

Figure 1(d). MBR of data

\subsection{CALCULATING OF PARKING SPOT}

2.2.1 Minimum parallel parking space calculating: Parking is a reversible process in this paper that means autonomous vehicle can drive out of the parking space according to the original track after parked into the parking space. The size of minimum parking spot is decided by kinematic constraint conditions and vehicle-crashed restrictions.

Figure 2 shows the sketch of parallel parking. Points abcd represent the autonomous car and $\mathrm{ABCD}$ represent the parked car. The point $S$ is located at the center of the rear axle. Another center of rear axle is $\mathrm{T}$ when the advancement direction of autonomous vehicle is $45^{\circ}$ relative to the $\mathrm{X}$-axis. And $\mathrm{E}$ is the point when the car parallel to $\mathrm{X}$-axis. So the curve STE means the track of vehicle abcd. When the car abcd drive out form the parking spot according to the track STE, and just collide with the car ABCD at the point $\mathrm{B}$. The coordinates of B is equal to the length $L_{p}$ and width $W_{p}$ of the minimum size of parallel parking.

So the size of minimum parallel parking spot can be extracted by the following formula:

$$
\begin{gathered}
L_{p}=y_{B}=W+2 l_{s} \\
W_{p}=x_{B}=\min \left(x_{B 1}, x_{B 2}\right) \\
x_{B 1}=L_{r o}+l_{s}+\sqrt{\left(2 R_{\min }-l_{s}\right) *\left(W+l_{s}\right)+\left(L-L_{r o}\right)^{2}} \\
x_{B 2}=L_{r o}+l_{s}+\sqrt{2} R_{\text {min }}-\sqrt{\left[(2-\sqrt{2}) R_{\text {min }}-W-l_{s}\right]\left[\sqrt{2} R_{\text {min }}+l_{s}\right]}
\end{gathered}
$$

Were $W=$ the width of the vehicle $L=$ The length of the vehicle $L_{\text {ro }}=$ Front overhang $\quad L_{f o}=$ Rear overhang $R_{\min }=$ Minimum turn radius of vehicle $L_{w b}=$ wheelbase $\quad L_{f}=$ Front gauge $L_{r}=$ Rear gauge

$l_{s}=$ Safe distance between vehicle and the boundary of parking spot

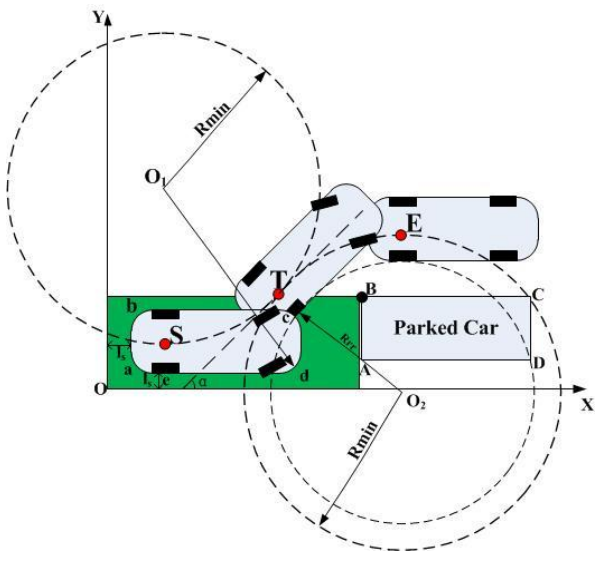

Figure 2. Minimum size of parallel parking

2.2.2 Minimum perpendicular parking space calculating: Perpendicular parking can divide into two situations according to the space in front of the parking spot. Figure 3(a) shows the situation when the parking space is enough. The size of minimum perpendicular parking spot can be extracted by the following formula:

$$
\begin{gathered}
L_{p 1}=\mathrm{L}+l_{s} \\
W_{p 1}=\mathrm{W}+2 l_{s}
\end{gathered}
$$

And Figure 3(b) represent the insufficient parking space situation. In this situation, the condition of collision constraints can be described as follow: when the car abcd drive out form the parking spot, the right rear wheel collide with the parked car at the point $\mathrm{B}$. and the left rear wheel collide with the parked car at the point point $\mathrm{C}$.

In this case, the following formula can be used:

$$
\begin{gathered}
L_{p 2}=\mathrm{L}+l_{s} \\
W_{p 2}=x_{C}-x_{B}=\sqrt{\left(R_{\text {min }}+\frac{W}{2}\right)^{2}+L_{r o}^{2}}-\frac{2 \sqrt{2} R_{\min }-W}{4}
\end{gathered}
$$




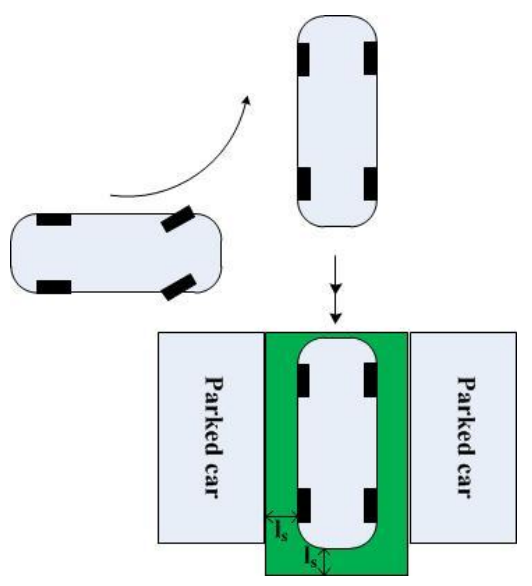

Figure 3(a). Sufficient parking space

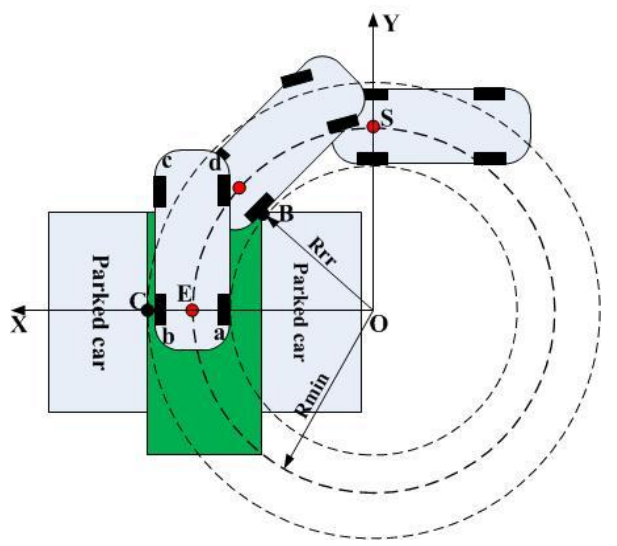

Figure 3(b). Insufficient parking space

\section{PATH PLANNING}

Rapidly-exploring Random Tree (RRT) is a fast and probabilistic algorithm which generally applied to robot path planning (Lavalle and Kuffner 2002). The basic RRT algorithm build the random tree to the global space at first, and then select a path as the planned path in the random tree. This way can explore the unknown area effectively. However the efficiency of this algorithm is low. In this paper, we improved it based on the moving characteristic of autonomous car.

As Figure4 show, this paper improved the RRT algorithm(Kalisiak and Panne 2006) in two aspects. The maximum turning angle $\theta_{\max }=70^{\circ}$ is used to constrain the space of new random points. And the distance between random points and the target points is a reference factor to improve the approaching speed of random-dot to target-dot. Besides, before the collision detection between autonomous vehicle and obstacles in the new random-dot, it is necessary to acquire the advancement direction of autonomous vehicle in the new random-dot. Like the following formula depicts, the new direction of vehicle is the average value between initial value and the value from initial point to new random point.

$\operatorname{azimuth}\left(x_{\text {new }}\right)=\left[\operatorname{azimuth}\left(x_{\text {init }}\right)+\operatorname{azimuth}\left(x_{\text {init }}, x_{\text {new }}\right)\right] / 2$

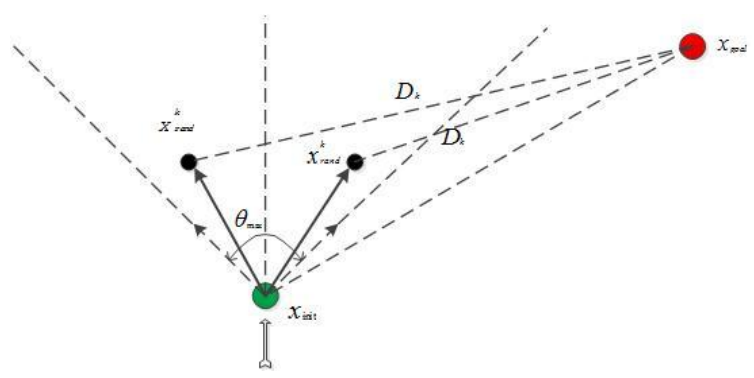

Figure 4. Selection of random point

\subsection{Path planning of parallel parking}

After detecting the effective parking spot, autonomous vehicle need to find the start point to drive into the parking spot. When the car drive to the start point, the body of car should parallel to the long side of the parking spot, and then start to park to the spot followed by two arc which consisted by two minimum turning radius.

As Figure 5 depict, $\mathrm{E}$ is the start point, and $\mathrm{S}$ is the parking spot. The coordinates of $\mathrm{S}$ and $\mathrm{E}$ can be described as follow:

$$
\begin{aligned}
& x_{S}=L_{r o}+l_{s} \\
& y_{S}=W_{p} / 2 \\
& x_{E}=\sqrt{2} R_{\text {min }}+x_{S} \\
& y_{E}=W_{p}+y_{S}
\end{aligned}
$$

In order to get into a right position, the starting place need to add L. So the coordinates of start point is $\left(\sqrt{2} R_{\min }+x_{S}+L, W_{p}+\right.$ $\left.y_{S}\right)$.

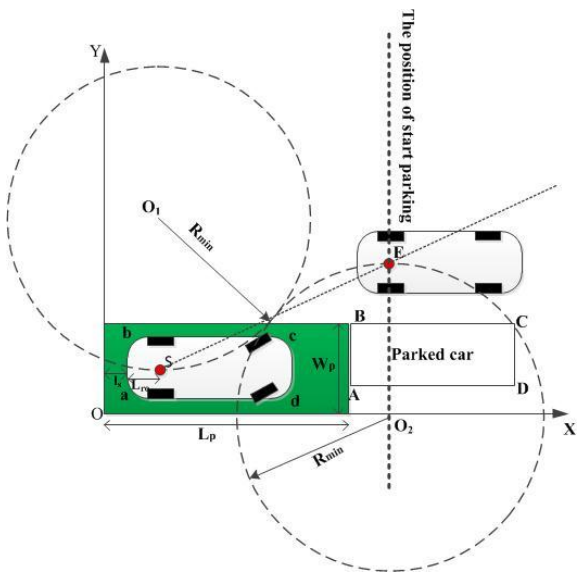

Figure 5. Path planning of parallel parking

Figure 6 shows the planned parking path with improved RRT algorithm. The white points stand for the accessible path, and the yellow points represent the start point and parking point. 


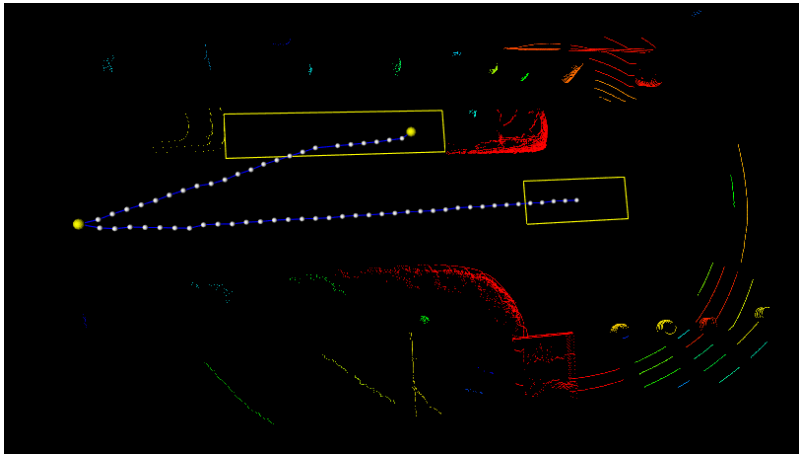

Figure 6. The result of path planning

\subsection{Path planning of perpendicular parking}

As Figure 7 show, $R_{r r}$ is the radius of trajectory. $L_{z}$ means the direct distance of parking in parking place. The coordinates of start point, parking point and $L_{z}$ can be described as follow:

$$
\begin{aligned}
& x_{S}=W_{p} / 2 \\
& y_{S}=L_{r o}+l_{s} \\
& x_{E}=R_{\text {min }}+x_{S} \\
& y_{E}=R_{\text {min }}+L_{z}+y_{S} \\
& L_{z}=L_{p}-l_{s}-L_{r o}-\frac{\sqrt{2} R_{r r}}{2}
\end{aligned}
$$

Also we add $\mathrm{L}$ to increase the space for getting into a right position. So the coordinates of start point is $\left(R_{\min }+x_{S}+\right.$ $\left.L, W_{p}+y_{S}\right)$.

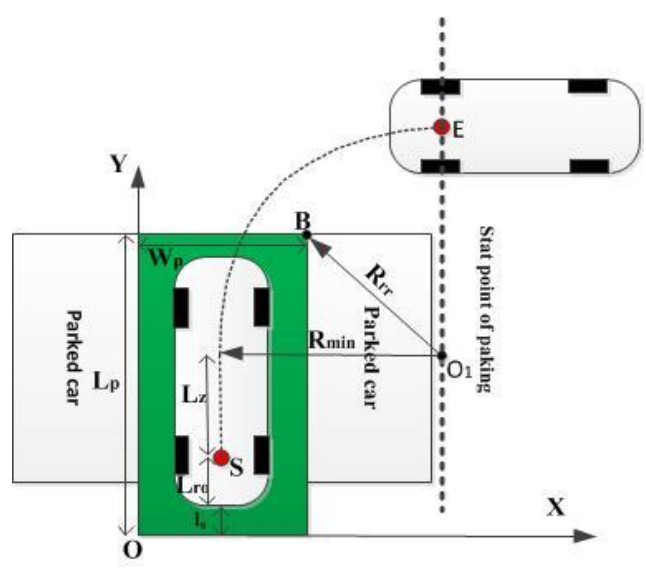

Figure 7. Path planning of perpendicular parking

Figure 8 shows the planned parking path of perpendicular parking.

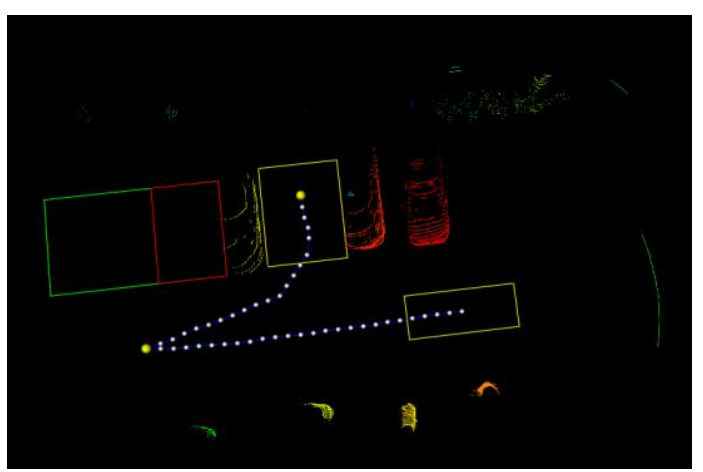

Figure 8 . The result of perpendicular parking

\section{CONTROL}

In order to control the speed of autonomous vehicle stability, the fuzzy logic controller(Zhao and Jr 2005) is designed to control the brake and accelerator.

\subsection{Fuzzy logic controller of brake}

The acceleration and the speed difference between the current and expect speed are input variables of fuzzy logic controller. And the two variables are fuzzed by trapezium membership functions.

The sugeno model is used in this paper to get the output data of brake intuitive. It is a nonlinear model for which is convenient to express the dynamic characteristics of complex system. The rulebase of brake is showed in table 2. Figure 9 depicts the specific output data of brake.

\begin{tabular}{|c|c|c|c|}
\hline $\begin{array}{c}\text { Speed } \\
\text { difference }\end{array}$ & $\begin{array}{c}\text { Acceleratio } \\
\mathrm{n}\end{array}$ & $\begin{array}{c}\text { Output controlled } \\
\text { data of brake }\end{array}$ & Value \\
\hline NS & $\mathrm{M}$ & $\mathrm{S}$ & 0 \\
\hline M & $\mathrm{M}$ & $\mathrm{MS}$ & 40 \\
\hline M & $\mathrm{N}$ & $\mathrm{S}$ & 0 \\
\hline M & $\mathrm{P}$ & $\mathrm{MS}$ & 40 \\
\hline PS & $\mathrm{M}$ & $\mathrm{MS}$ & 40 \\
\hline PS & $\mathrm{N}$ & $\mathrm{S}$ & 0 \\
\hline PS & $\mathrm{P}$ & $\mathrm{MS}$ & 40 \\
\hline PB & $\mathrm{M}$ & $\mathrm{MB}$ & 60 \\
\hline PB & $\mathrm{N}$ & $\mathrm{MS}$ & 40 \\
\hline PB & $\mathrm{P}$ & $\mathrm{B}$ & 70 \\
\hline
\end{tabular}

Table 2. Rulebase of brake

Where $\quad \mathrm{NS}=$ negative small; $\mathrm{M}=$ middle $\mathrm{PS}=$ positive small; $\mathrm{PB}=$ positive $\mathrm{big}$ $\mathrm{N}=$ Negative; $\mathrm{P}=$ positive $\mathrm{S}=$ small $; \mathrm{MS}=$ middle small $\mathrm{MB}=$ middle big; $\mathrm{B}=$ big

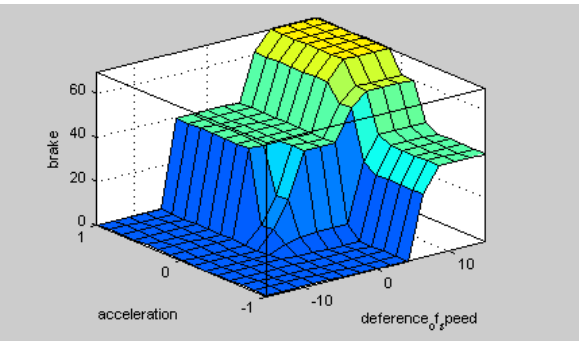

Figure 9. Output data of brake 


\subsection{Fuzzy logic controller of accelerator:}

The membership function of accelerator is different from brake. The rulebase of accelerator is showed in table 3 . Figure 10 depicts the specific output data of accelerator.

\begin{tabular}{|c|c|c|c|}
\hline $\begin{array}{c}\text { Speed } \\
\text { difference }\end{array}$ & Acceleration & $\begin{array}{c}\text { Output controlled } \\
\text { data of accelerator }\end{array}$ & Value \\
\hline NS & M & MS & 150 \\
\hline NS & N & MB & 170 \\
\hline NS & P & MS & 150 \\
\hline M & M & S & 0 \\
\hline M & N & MS & 150 \\
\hline M & P & MS & 150 \\
\hline PS & & S & 0 \\
\hline NB & M & MB & 170 \\
\hline NB & N & B & 220 \\
\hline NB & P & MB & 170 \\
\hline
\end{tabular}

Table 3. Rulebase of accelerator

Where

$\mathrm{NB}=$ Negative big; $\mathrm{NS}=$ Negative small $\mathrm{M}=$ middle; $\mathrm{PS}=$ positive small

$\mathrm{N}=$ Negative; $\mathrm{P}=$ positive

$\mathrm{S}=$ small $; \mathrm{MS}=$ middle small

$\mathrm{MB}=$ middle big; $\mathrm{B}=$ big

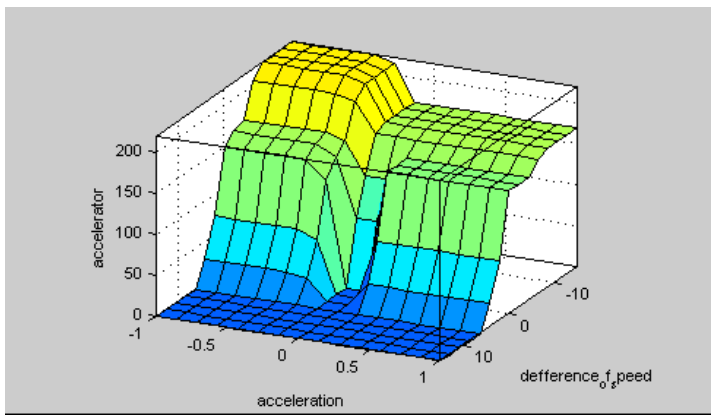

Figure 10. Output data of brake

\section{EXPERIMENTAL IMPLEMENTATION}

The algorithm was implemented on "TuZhi" autonomous vehicle of Wuhan University. The vehicle is equipped with a suite of sensors including velodyne-32E, GPS, IMU and a wheel encoder etc.

\subsection{Experimental of parallel parking}

Figure 11 shows the processing of parallel parking. Figure 11(a) shows the position of vehicle after it detected the efficient parking spot. And inferred the situation between parallel parking and perpendicular parking by the posture of parked cars. The position of vehicle showed in Figure 11(b) is the start parking point. Figure 11(c) shows the process of autonomous parking. And Figure 11(d) shows the vehicle stopped in parking spot.
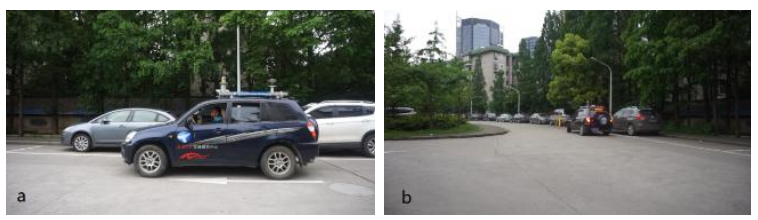
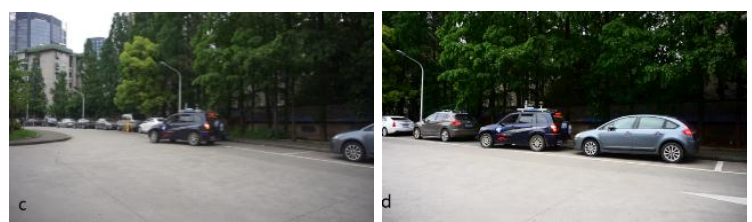

Figure 11. The processing of parallel parking

Figure 12 shows the error of actual path and planned path of parking (green),and the red curve is planned path. From Figure 13 we can tell that the maximum error is about $10 \mathrm{~cm}$, and when stopped in the parking spot, the final error is about $5 \mathrm{~cm}$. Figure 14 expresses the difference between the actual and specified speed of vehicle.

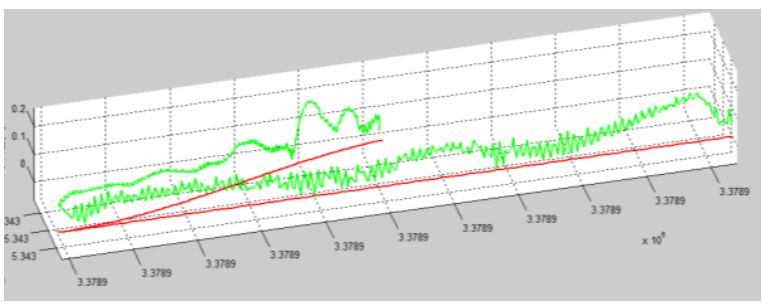

Figure 12. The error of path

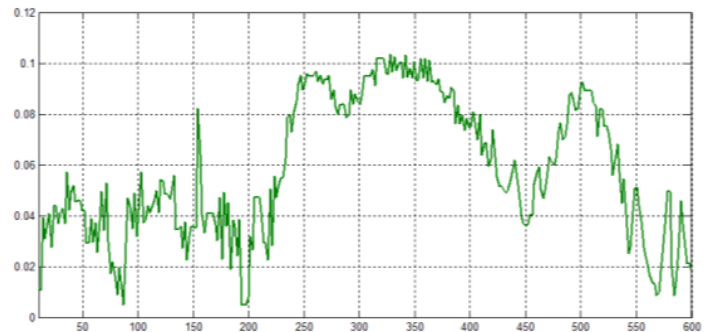

Figure 13. Specific difference of path

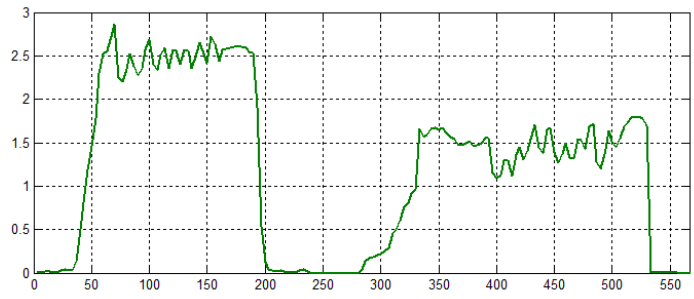

Figure 14. Difference between speed

\subsection{Experimental of perpendicular parking}

Figure 15 shows the processing of perpendicular parking. It is similar to parallel parking. Results show that it need 30 s to parking the autonomous vehicle. And the error of actual and planned path of perpendicular parking (green) showed in Figure 16. Figures 17 and 18 show values of error and difference of speed.
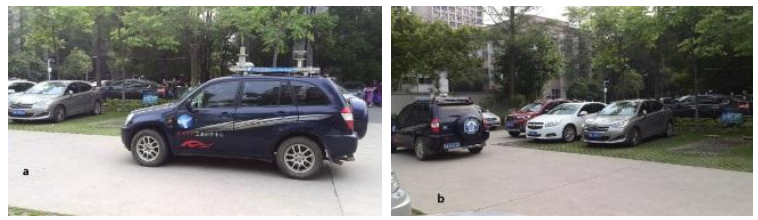

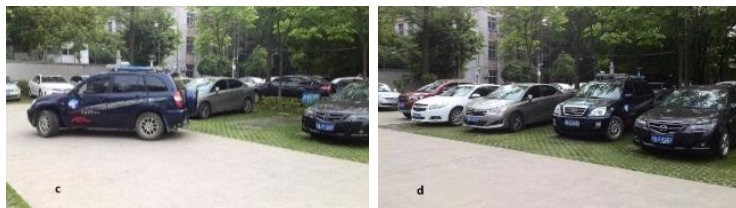

Figure 15. The processing of perpendicular parking

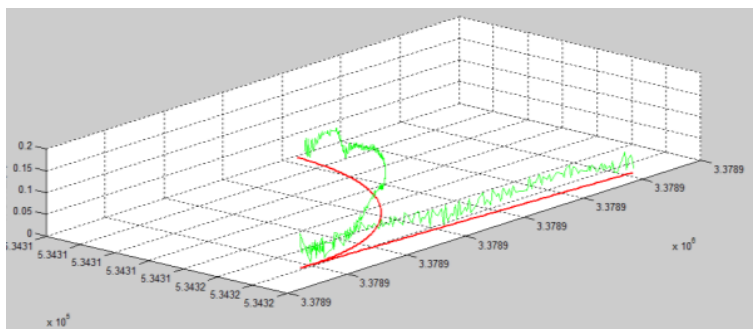

Figure 16. The error of path

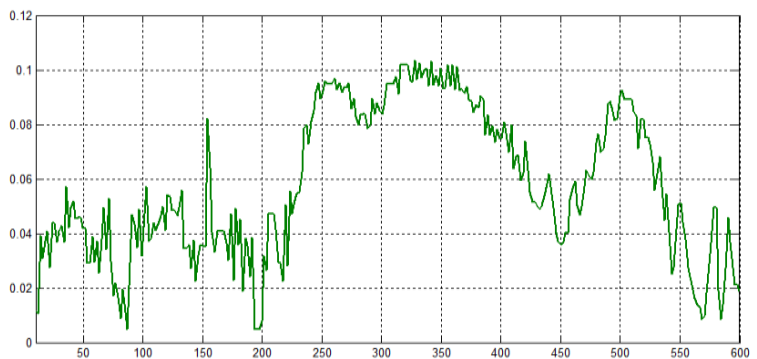

Figure 17. Specific difference of path

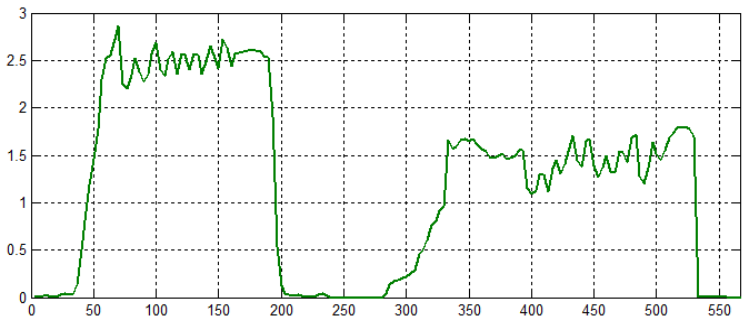

Figure 18. Difference between speed

\section{CONCLUSION}

This paper has proposed an automatic parking system based on self-driving vehicle. The start and parking point have been calculated based on the vehicle dynamic model. And have improved the RRT algorithm to plan the parking path. Furthermore two fuzzy logic controller have been designed to control the brake and accelerator to realize the steady of speed. From the results obtained, it can be seen that the automatic system is able to park the autonomous vehicle safe and fast.

Future works include the following: 1) eliminating the near blind area of LiDAR to improve the ability of environment perception. 2) Adding the camera data to recognize the white parking line to improve the ability of parking detection.

\section{REFERENCES}

Wang, W., et al, 2014. Automatic parking of vehicles: A review of literatures. International Journal of Automotive Technology 15(6), pp. 967-978.

Jung, H. G., et al, 2008. Scanning Laser Radar-Based Target Position Designation for Parking Aid System. IEEE Transactions on Intelligent Transportation Systems 9(3), pp. 406-424.

Park, W. J., et al, 2008. Parking space detection using ultrasonic sensor in parking assistance system. Intelligent Vehicles Symposium. pp. 1039-1044. IEEE.

Kaempchen, N., et al, 2002. Stereo vision based pose estimation of parking lots using 3D vehicle models. Intelligent Vehicle Symposium. pp.459-464. IEEE.

Marouf, M., et al, 2014 Automatic parallel parking and platooning to redistribute electric vehicles in a car-sharing application. Intelligent Vehicles Symposium Proceedings. pp.486-491. IEEE.

Laugier, C. and I. E. Paromtchik, 1996. Autonomous parallel parking of a nonholonomic vehicle. Intelligent Vehicles Symposium, 1996. pp.13 - 18. IEEE.

Zhao, Y. and E. G. C. Jr, 2005. Robust automatic parallel parking in tight spaces via fuzzy logic. Robotics \& Autonomous Systems 51(2). pp. 111-127.

Yang, X. and F. You, 2010. Hierarchical Variable Universe Fuzzy Controller for Truck Backer Upper Problem. Fuzzy Systems and Mathematics 24(2). pp. 152-156.

Min, K. and J. Choi, 2013. A Control System for Autonomous Vehicle. Valet Parking. pp. 1714-1717.

Liu, K. Z., et al, 2005. Theory and experiments on automatic parking systems. Control, Automation. Robotics and Vision Conference, 2004. pp. 861-866 Vol. 2.

Song, J, 2009. The key technical research of autonomous parking system. National University of Defense Technology. 38(2). pp. 225-228.

Lavalle, S. M. and J. J. Kuffner, 2002. Report on the International Conference on Robotics and Automation Workshop. "Randomized kinodynamic planning". Vol.1, pp.473-479. IEEE.

Kalisiak, M. and M. V. D. Panne, 2006. Report on the International Conference on Robotics and Automation Workshop. "RRT-blossom: RRT with a local flood-fill behavior". pp.12371242. IEEE. 